Bull. Korean Math. Soc. 51 (2014), No. 6, pp. 1591-1603

http://dx.doi.org/10.4134/BKMS.2014.51.6.1591

\title{
MAXIMUM PRINCIPLE, CONVERGENCE OF SEQUENCES AND ANGULAR LIMITS FOR HARMONIC BLOCH MAPPINGS
}

\author{
JinJing QiaO AND Hongya GaO
}

\begin{abstract}
In this paper, we investigate maximum principle, convergence of sequences and angular limits for harmonic Bloch mappings. First, we give the maximum principle of harmonic Bloch mappings, which is a generalization of the classical maximum principle for harmonic mappings. Second, by using the maximum principle of harmonic Bloch mappings, we investigate the convergence of sequences for harmonic Bloch mappings. Finally, we discuss the angular limits of harmonic Bloch mappings. We show that the asymptotic values and angular limits are identical for harmonic Bloch mappings, and we further prove a result that applies also if there is no asymptotic value. A sufficient condition for a harmonic Bloch mapping has a finite angular limit is also given.
\end{abstract}

\section{Preliminaries}

A complex-valued function $f$ is said to be harmonic in a simply connected domain $\Omega$ of the complex plane $\mathbb{C}$ if and only if both $\operatorname{Re}\{f\}$ and $\operatorname{Im}\{f\}$ are real harmonic mappings in $\Omega$. Every harmonic mapping $f$ in $\Omega$ has the canonical decomposition:

$$
f=h+\bar{g},
$$

where both $h$ and $g$ are analytic in $\Omega$ and $g\left(z_{0}\right)=0$ for some prescribed point $z_{0} \in \Omega$ (cf. [8]). Let $\mathbb{D}=\{z \in \mathbb{C}:|z|<1\}$ and $\mathbb{T}=\{z:|z|=1\}$. Throughout this paper, we consider harmonic mappings in $\mathbb{D}$.

Definition 1. Let $f$ be a harmonic mapping satisfying the Lipschitz condition when regarded as a function from the hyperbolic disk $\mathbb{D}$ into $\mathbb{C}$, endowed with

Received July 16, 2013; Revised April 6, 2014.

2010 Mathematics Subject Classification. Primary 30C65, 30C45; Secondary 30C20.

Key words and phrases. harmonic Bloch mapping, maximum principle, convergence, angular limit.

The first author was supported by NSF of Hebei Science Foundation for Young Scientists (No. A2013201104). The second author was supported by NSF of Hebei Province (No. A2011201011). 
the Euclidean distance. The function $f$ is called a harmonic Bloch mapping and the Lipschitz number

$$
\sup _{z \neq w} \frac{|f(z)-f(w)|}{\rho(z, w)}
$$

is called the Bloch constant of $f$, where $\rho$ denotes the hyperbolic distance in $\mathbb{D}$, that is,

$$
\rho(z, w)=\frac{1}{2} \log \left(\frac{1+r}{1-r}\right)
$$

and $r$ is the modulus of $\frac{z-w}{1-\bar{z} w}$ (cf. [6]).

For an analytic function $f$, its Bloch constant is (see [7, Theorem 10])

$$
\sup _{z \neq w} \frac{|f(z)-f(w)|}{\rho(z, w)}=\sup _{z \in \mathbb{D}}\left(1-|z|^{2}\right)\left|f^{\prime}(z)\right| .
$$

See, for example, [1] for more details.

As a generalization, in [6], the author proved that the Bloch constant of a harmonic mapping $f=h+\bar{g}$ has the following expression:

$$
\sup _{z \neq w} \frac{|f(z)-f(w)|}{\rho(z, w)}=\sup _{z \in \mathbb{D}}\left(1-|z|^{2}\right)\left(\left|h^{\prime}(z)\right|+\left|g^{\prime}(z)\right|\right) .
$$

Obviously, a function $f=h+\bar{g}$ is Bloch if and only if both $h$ and $g$ are Bloch.

In the following, we introduce some necessary notions and notations.

Let $\mathscr{B}_{\mathcal{H}}$ (resp. $\mathscr{B}$ ) denote the class of all harmonic mappings (resp. analytic functions) $f$ with $\sup _{z \in \mathbb{D}}\left(1-|z|^{2}\right)\left(\left|h^{\prime}(z)\right|+\left|g^{\prime}(z)\right|\right)<\infty\left(\operatorname{resp} . \sup _{z \in \mathbb{D}}(1-\right.$ $\left.\left.|z|^{2}\right)\left|f^{\prime}(z)\right|<\infty\right)$. It is easy to see that $\mathscr{B}_{\mathcal{H}}($ resp. $\mathscr{B})$ is a Banach space with the norm

$$
\begin{gathered}
\|f\|_{\mathscr{B}_{\mathcal{H}}}=|f(0)|+\sup _{z \in \mathbb{D}}\left(1-|z|^{2}\right)\left(\left|h^{\prime}(z)\right|+\left|g^{\prime}(z)\right|\right) \\
\text { (resp. } \left.\|f\|_{\mathscr{B}}=|f(0)|+\sup _{z \in \mathbb{D}}\left(1-|z|^{2}\right)\left|f^{\prime}(z)\right|\right),
\end{gathered}
$$

which is called the harmonic Bloch space (resp. Bloch space). Each element in $\mathscr{B}_{\mathcal{H}}$ (resp. $\left.\mathscr{B}\right)$ is a harmonic Bloch mapping (resp. Bloch function).

The little harmonic Bloch space $\mathscr{B}_{\mathcal{H}, 0}$ (resp. little Bloch space $\mathscr{B}_{0}$ ) is the set of all mappings $f \in \mathscr{B}_{\mathcal{H}}$ (resp. $f \in \mathscr{B}$ ) satisfying

$$
\left.\lim _{|z| \rightarrow 1}\left(1-|z|^{2}\right)\left(\left|h^{\prime}(z)\right|+\left|g^{\prime}(z)\right|\right)=0 \text { (resp. } \lim _{|z| \rightarrow 1}\left(1-|z|^{2}\right)\left|f^{\prime}(z)\right|=0\right) .
$$

Each element in $\mathscr{B}_{\mathcal{H}, 0}$ (resp. $\mathscr{B}_{0}$ ) is called a little harmonic Bloch mapping (resp. little Bloch function).

Definition 2. We say that the harmonic mapping $f$ in $\mathbb{D}$ has the asymptotic value $a \in \mathbb{C}$ at the point $\xi \in \mathbb{T}$ if there exists a Jordan arc $\Gamma$ that ends at $\xi$ and lies otherwise in $\mathbb{D}$ such that

$$
f(z) \rightarrow a \text { for } z \in \Gamma, z \rightarrow \xi .
$$

We call such an arc an asymptotic path. If $\Gamma=\{\xi r: 0 \leq r \leq 1\}$, we call $a$ a radial limit (cf. [10]). 
Definition 3. A (symmetric) Stolz angle is a set of the form

$$
A=\left\{z \in \mathbb{D}:|\arg (1-\bar{\xi} z)|<\frac{\pi}{2}-\delta\right\} \quad\left(0<\delta<\frac{\pi}{2}\right),
$$

that is a sector of vertex $\xi$ and angle less than $\pi$ symmetric to $[0, \xi]$. We say that $f$ has the angular limit $a$ at $\xi \in \mathbb{T}$ if

$$
f(z) \rightarrow a \text { as } z \rightarrow \xi, z \in A
$$

for every Stolz angle $A$ at $\xi$ (cf. [10]).

It is well known that (analytic) Bloch functions are normal functions (see [10, p. 261] for definition of normal functions). In this paper, we consider properties of harmonic Bloch mappings, which are generalizations of the corresponding ones for normal functions or Bloch functions.

In [9] (see also [10, Theorem 9.1]), it is given the maximum principle for normal functions, which is a generalization of the classical maximum principle for analytic functions since there is no any assumption on $|f(z)|$ with $z$ belonging to some subarc of the boundary. In Section 2, we consider the maximum principle of harmonic Bloch mappings, and get a harmonic analog of [10, Theorem 9.1], which is a generalization of the classical maximum principle for harmonic mappings.

In Section 3, we consider the convergence for a sequence of harmonic Bloch mappings. By using maximum principle of harmonic Bloch mappings which is obtained in Section 2, we prove that a sequence of harmonic Bloch mappings $f_{n}$ converges to 0 as $n \rightarrow \infty$ in the unit disk under the condition that $\max _{z \in C_{n}}\left|f_{n}(z)\right|$ converges to 0 , where $\left\{C_{n}\right\}$ is a sequence of closed Jordan arcs with positive measure. Our main result is Theorem 2, which is a generalization of [10, Theorem 9.2] for normal functions.

Finally, in the last section, we consider the angular limits of harmonic Bloch mappings. We first show that the asymptotic values and angular limits are identical for harmonic Bloch mappings. Then, as a generalization of [10, Theorem 9.5], by using different method, we prove that for a set $E$, if $\sup _{z \in \mathbb{D}}|\operatorname{Im} g(z)|<\infty$ or $\sup _{z \in \mathbb{D}}|\operatorname{Im} h(z)|<\infty$, limsup $\operatorname{dist}[f(z), E]$ exists as $z \rightarrow \xi \in \mathbb{T}$ along some arc $\Gamma \subset \mathbb{D}$ with $\xi \in \Gamma$, then this is true in every Stolz angle. A sufficient condition for a harmonic Bloch mapping has a finite angular limit is also given.

\section{Maximum principle for harmonic Bloch mappings}

Before giving the main result, we give the following lemma which will be useful in the proof of the main result.

Lemma 1. Let $\varphi$ be analytic in $\mathbb{D}$ and $|\varphi(z)|<1$. If $f=h+\bar{g}$ is a harmonic Bloch mapping in $\mathbb{D}$ and

$$
\sup _{z \in \mathbb{D}}\left(1-|z|^{2}\right)\left(\left|h^{\prime}(z)\right|+\left|g^{\prime}(z)\right|\right)=\alpha<\infty,
$$


then $F=f \circ \varphi=H+\overline{H_{1}}$ is also Bloch in $\mathbb{D}$, and

$$
\sup _{z \in \mathbb{D}}\left(1-|z|^{2}\right)\left(\left|H^{\prime}(z)\right|+\left|H_{1}^{\prime}(z)\right|\right) \leq \alpha,
$$

with equality if $\varphi$ is a Möbius transformation of $\mathbb{D}$ onto itself.

Proof. By G. Pick's Theorem ([3, p. 6]), we have

$$
\left(1-|z|^{2}\right)\left|\varphi^{\prime}(z)\right| \leq 1-\left|\varphi^{\prime}(z)\right|^{2},
$$

with equality for a Möbius transformation of $\mathbb{D}$ onto itself, it follows that

$$
\begin{aligned}
\left(1-|z|^{2}\right)\left(\left|H^{\prime}(z)\right|+\left|H_{1}^{\prime}(z)\right|\right) & =\left(1-|z|^{2}\right)\left|\varphi^{\prime}(z)\right|\left(\left|h^{\prime}(\varphi(z))\right|+\left|g^{\prime}(\varphi(z))\right|\right) \\
& \leq\left(1-|\varphi(z)|^{2}\right)\left(\left|h^{\prime}(\varphi(z))\right|+\left|g^{\prime}(\varphi(z))\right|\right) \\
& \leq \alpha .
\end{aligned}
$$

The proof of the lemma is complete.

Theorem 1. Let $f=h+\bar{g} \in \mathscr{B}_{\mathcal{H}}$ and

$$
\sup _{z \in \mathbb{D}}\left(1-|z|^{2}\right)\left(\left|h^{\prime}(z)\right|+\left|g^{\prime}(z)\right|\right) \leq \alpha<\infty .
$$

Let $G$ be a domain with $\bar{G} \subset \mathbb{D}$ that lies in the lens-shaped domain of angle $\beta$ $(0<\beta<\pi)$ cut off from $\mathbb{D}$ by the circular arc $B$. We suppose that

$$
|f(z)| \leq \delta
$$

for $z \in \partial G \backslash B$. Then

$$
|f(z)| \leq \eta
$$

for $z \in G$, where $\eta=\eta(\delta, \alpha, \beta)$ is the smallest positive solution of

$$
\delta=\eta \exp \left[-\frac{\kappa}{2 \eta}\right]
$$

with $\kappa=\alpha \beta / \sin \beta$.

This theorem is a generalization of the classical maximum principle for harmonic mappings in so far as it does not make any assumption about $|f(z)|$ for $z \in B \cap \partial G$. On the other hand, this theorem is highly non-linear: It is only when, compared to $1 / \alpha,|f(z)|$ is rather small on $\partial G \backslash B$ that we get any estimate at all.

Since the function $t \exp \left[-\frac{\kappa}{2 t}\right]$ is increasing for $0<t<\infty$, it follows that, for $\delta \geq 0,(2.4)$ has a unique solution $\eta$ with $\eta \geq 0$.

This theorem is also a generalization of [10, Theorem 9.1] for analytic functions which is a basic result for the maximum principle for normal functions obtained by Lehto and Virtanen in [9].

Proof of Theorem 1. By choosing a suitable Möbius transformation $\varphi$ of $\mathbb{D}$ onto $\mathbb{D}$ and replacing $f$ by $F=f \circ \varphi$, we assume that $B$ is a circular arc passing 


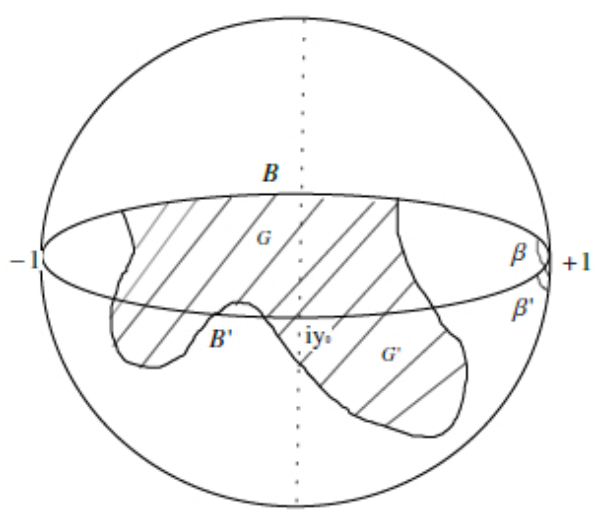

FIGURE 1.

through -1 and 1 , and $G$ lies below the arc $B$. By Lemma $1, F=f \circ \varphi=H+\overline{H_{1}}$ is also Bloch in $\mathbb{D}$, and

$$
\sup _{z \in \mathbb{D}}\left(1-|z|^{2}\right)\left(\left|H^{\prime}(z)\right|+\left|H_{1}^{\prime}(z)\right|\right) \leq \alpha .
$$

For $0<\beta^{\prime}<\beta$, let $G^{\prime}$ be the intersection of $G$ with the domain of angle $\beta^{\prime}$ cut off by the circular arc $B^{\prime}$ through \pm 1 (see Figure 1).

Suppose that (2.3) does not hold. Since $|f(z)| \leq \delta<\eta(z \in \partial G \backslash B)$ by (2.2) and (2.4), and since $\bar{G} \subset \mathbb{D}$, there exists $\beta^{\prime}$ such that $|f(z)| \leq \eta$ for $z \in G^{\prime}$. Let $\beta^{\prime}\left(0<\beta^{\prime}<\beta\right)$ be the largest such number. Then

$$
\eta=\sup _{z \in G^{\prime}}|f(z)|=\left|f\left(z_{0}\right)\right|
$$

for some $z_{0} \in B^{\prime} \backslash \partial G$. By a further Möbius transformation we may assume that $z_{0}=i y_{0},-1<y_{0}<1$, where

$$
y_{0}=\tan \left(\frac{\beta^{\prime}}{2}-\frac{\pi}{4}\right)
$$

Let

$$
a(z)=|f(z)| \exp \left[\frac{b}{i} \log \frac{1+z}{1-z}+\frac{\pi b}{2}-2 b \beta^{\prime}\right] \quad\left(z \in G^{\prime}\right),
$$

where $b=\frac{1}{\beta^{\prime}} \log \frac{\eta}{\delta}(>0)$. It is known that every point in $\mathbb{D}$ is on one circular arc that passes through -1 , iy and 1 for some $-1<y<1$, and on this circular $\operatorname{arc} \arg [(1+z) /(1-z)]=\arg [(1+i y) /(1-i y)]=2 \arctan y$, it follows that $\exp \left[\frac{b}{i} \log \frac{1+z}{1-z}+\frac{\pi b}{2}-2 b \beta^{\prime}\right]$ and $|a(z)|$ with $z \in \overline{G^{\prime}}$ attains its maximum on the boundary point $z_{0}=i y_{0}$. Since $\arg [(1+z) /(1-z)]=\beta^{\prime}-\frac{\pi}{2}$ for $z \in B^{\prime}$, it follows from (2.5) that

$$
\max _{z \in B^{\prime} \cap \partial G^{\prime}}|a(z)| \leq \eta \exp \left(-b \beta^{\prime}\right)=\delta .
$$


Since $\partial G^{\prime} \backslash B^{\prime} \subset \partial G \backslash B$, we obtain from (2.2) that

$$
\sup _{z \in \partial G^{\prime} \backslash B^{\prime}}|a(z)| \leq \sup _{z \in \partial G^{\prime} \backslash B^{\prime}}|f(z)| \leq \sup _{z \in \partial G \backslash B}|f(z)| \leq \delta .
$$

Hence from the property that $|a(z)|$ with $z \in \overline{G^{\prime}}$ attains its maximum on the boundary point $z_{0}=i y_{0}$, it follows that $|a(z)| \leq \delta$ for $z \in G^{\prime}$, and then

$$
\log |f(i y)| \leq \log \delta+2 b \beta^{\prime}-\frac{\pi b}{2}-2 b \arctan y, \quad i y \in G^{\prime} .
$$

We have from (2.6) that $\beta^{\prime}=\frac{\pi}{2}-2 \arctan y_{0}$. Since $\left|f\left(i y_{0}\right)\right|=\eta$, we have

$$
\log \left|f\left(i y_{0}\right)\right|=\log \eta=\log \delta+b \beta^{\prime}=\log \delta+2 b \beta^{\prime}-\frac{\pi b}{2}-2 b \arctan y_{0} .
$$

Therefore

$$
\log |f(i y)|-\log \left|f\left(i y_{0}\right)\right| \leq-2 b\left(\arctan y-\arctan y_{0}\right), i y \in G^{\prime} .
$$

Letting $y \rightarrow y_{0}^{+}$yields that

$$
\operatorname{Re}\left[i \frac{h^{\prime}\left(i y_{0}\right)-\overline{g^{\prime}}\left(i y_{0}\right)}{f\left(i y_{0}\right)}\right] \leq \frac{-2 b}{1+y_{0}^{2}}=-\frac{2 \log (\eta / \delta)}{\beta^{\prime}\left(1+y_{0}^{2}\right)} .
$$

On the other hand, since $\left|f\left(i y_{0}\right)\right|=\eta$, it follows from (2.1) that

$$
\left|\frac{h^{\prime}\left(i y_{0}\right)-\overline{g^{\prime}}\left(i y_{0}\right)}{f\left(i y_{0}\right)}\right| \leq \frac{\left|h^{\prime}\left(i y_{0}\right)\right|+\left|g^{\prime}\left(i y_{0}\right)\right|}{\left|f\left(i y_{0}\right)\right|} \leq \frac{\alpha}{\eta\left(1-y_{0}^{2}\right)}=\frac{\alpha}{\eta\left(1+y_{0}^{2}\right) \sin \beta^{\prime}} .
$$

Hence (2.7) and (2.8) imply that

$$
\delta \geq \eta \exp \left[-\frac{\kappa^{\prime}}{2 \eta}\right], \quad \kappa^{\prime}=\frac{\alpha \beta^{\prime}}{\sin \beta^{\prime}},
$$

which contradicts $(2.4)$ because $\kappa^{\prime}<\kappa$.

\section{The convergence for sequences of harmonic Bloch mappings}

We begin this section with the following lemma, which is identity theorem for harmonic mappings, see, for example, [5, Lemma 2].

Lemma 2. Let $f$ be harmonic in a connected open set $D$. If $f(z) \equiv 0$ in some open subset $G$ of $D$, then $f(z) \equiv 0$ in $D$.

Marty's normality criterion (see [10, p. 262]) states that a family of meromorphic functions is normal if and only if their spherical derivative (see [10, p. 261] for the definition of spherical derivative) are locally uniformly bounded. Hence a sequence of Bloch function is normal, and then a sequence of harmonic Bloch mappings is also normal since both the analytic part and anti-analytic part of a harmonic Bloch mapping are Bloch, this fact will be used in the proof of the following theorem. 
Theorem 2. Let $f_{n} \in \mathscr{B}_{\mathcal{H}}$, and

$$
\sup _{z \in \mathbb{D}}\left(1-|z|^{2}\right)\left(\left|h_{n}^{\prime}(z)\right|+\left|g_{n}^{\prime}(z)\right|\right) \leq \alpha<\infty, \quad n=1,2, \ldots
$$

If there exist closed Jordan arcs $C_{n} \subset \mathbb{D}$ such that

$$
\operatorname{diam} C_{n}=\sup _{z, w \in C_{n}}|z-w|>\gamma>0, \quad n=1,2, \ldots
$$

and

$$
\max _{z \in C_{n}}\left|f_{n}(z)\right| \rightarrow 0 \quad(n \rightarrow \infty),
$$

then $f_{n}(z) \rightarrow 0$ as $n \rightarrow \infty$, locally uniformly in $\mathbb{D}$.

Proof. Suppose that the assertion is false. By (3.1) and Marty's criterion, the sequence $\left\{f_{n}\right\}$ is normal in $\mathbb{D}$. Taking a subsequence we may therefore assume that

$$
f_{n}(z) \rightarrow f(z) \text { as } n \rightarrow \infty \text {, locally uniformly in } \mathbb{D} \text {, }
$$

where $f$ is a harmonic mapping such that $f\left(z_{0}\right) \neq 0$ for some $z_{0} \in \mathbb{D}$.

Now, we consider the first case that

$$
r_{n}=\inf \left\{|z|: z \in C_{n}\right\} \rightarrow 1 \quad(n \rightarrow \infty) .
$$

By (3.2), there exist points $a_{n}, b_{n} \in C_{n}$ with $\left|a_{n}-b_{n}\right|>\gamma$. If $B_{n}$ denotes the circle through $a_{n}$ and $b_{n}$ that is orthogonal to $\mathbb{T}$, then for $n$ is sufficiently large, $a_{n}$ and $b_{n}$ lie on different $\operatorname{arcs}$ of $B_{n}^{*}=B_{n} \cap\left\{r_{n} \leq\left|z_{n}\right| \leq 1\right\}$. Hence we can find a subarc $C_{n}^{\prime}$ of $C_{n}$ that intersects each arc of $B_{n}^{*}$ exactly once. By (3.5), the subarc $B_{n}^{\prime}$ of $B_{n}$ between the endpoints of $C_{n}^{\prime}$ does not intersect $C_{n}^{\prime}$ at any other point.

If $G_{n}$ is the inner domain of the Jordan curve $B_{n}^{\prime} \cup C_{n}^{\prime}$, then $\partial G_{n}=B_{n}^{\prime} \cup C_{n}^{\prime} \subset$ $\mathbb{D}$, which shows that $\overline{G_{n}} \subset \mathbb{D}$. Hence we obtain from (3.1), (3.3) and Theorem 1 (with $\beta=\frac{\pi}{2}$ ) that

$$
\max _{z \in B_{n}^{\prime}}\left|f_{n}(z)\right| \leq \max _{z \in \overline{G_{n}}}\left|f_{n}(z)\right| \rightarrow 0 \quad(n \rightarrow \infty) .
$$

Since $B_{n}^{\prime}$ intersects $\{|z| \leq r\}$ for some $r<1$ and large $n$, it therefore follows from (3.4) and Lemma 2 that $f(z) \equiv 0$, which is false.

In the case that (3.5) does not hold, $C_{n}$ intersects $\{|z| \leq r\}$ for some $r<1$ and infinitely many $n$. Hence it follows from (3.2), (3.3), (3.4) and Lemma 2 that $f(z) \equiv 0$, and this is false.

A sequence $\left\{C_{n}\right\}$ of closed Jordan $\operatorname{arcs} C_{n} \subset \mathbb{D}$ is called a sequence of harmonic Koebe arcs with respect to the harmonic mapping $f$ if $\operatorname{diam} C_{n} \geq$ $\gamma>0(n=1,2, \ldots)$ and if, for some $c \in \mathbb{C}$,

$$
\max _{z \in C_{n}}|f(z)-c| \rightarrow 0, n \rightarrow \infty .
$$

We say that $f$ has no Koebe arcs if no such sequence exists.

Corollary 1. A non-constant harmonic Bloch mapping has no Koebe arcs. 
This corollary is a generalization of [10, Corollary 9.1] for normal functions, which is obtained by Bagemihl and Seidel in 1961. The proof follows from Theorem 2 for $f_{n}(z) \equiv f(z)$, here we omit it.

Corollary 2. Let $f$ be non-constant harmonic Bloch in $\mathbb{D}$ and let $\Gamma: z(t)$, $0 \leq t<1$ be a half-open Jordan arc in $\mathbb{D}$ with $|z(t)| \rightarrow 1(t \rightarrow 1)$. If

$$
f(z(t)) \rightarrow a \text { as } t \rightarrow 1^{-}
$$

then $z(t) \rightarrow \xi\left(t \rightarrow 1^{-}\right)$for some $\xi \in \mathbb{T}$ so that $\Gamma$ is an asymptotic path.

Proof. It is clear that $z(t)$ has at least one limit point on $\mathbb{T}$ as $t \rightarrow 1^{-}$. Suppose there are two distinct limit points $\xi, \xi^{\prime} \in \mathbb{T}$. Then we can find closed subarcs $C_{n}$ of $\Gamma$ such that $\operatorname{diam} C_{n} \rightarrow\left|\xi-\xi^{\prime}\right| \neq 0$ as $n \rightarrow \infty$. By (3.6), they form a sequence of Koebe arcs, contrary to Corollary 1.

\section{The angular limit of harmonic Bloch mappings}

By Definition 2, an angular limit is a radial limit and therefore an asymptotic value. In the following theorem, we will show that the converse holds for harmonic Bloch mappings. Therefore a harmonic Bloch mapping has at most one asymptotic value at any given point $\xi \in \mathbb{D}$.

Theorem 3. If the harmonic mapping $f \in \mathscr{B}_{\mathcal{H}}$ has the asymptotic value a at $\xi$, then $f$ also has the angular limit a at $\xi$.

The main idea of the proof for this theorem comes from the proof of $[10$, Theorem 9.3] (see also [9]). For the convenience, we give the proof.

Proof of Theorem 3. Without loss of generality, we assume that $\xi=1$ and $a=0$. Suppose that $z_{n} \rightarrow 1$ for $z_{n} \in A$, where $A$ is a Stolz angle at $\xi$. First, we choose two real sequences $\left\{\xi_{n}\right\}$ and $\left\{y_{n}\right\}$, and $r<1$ such that

$$
z_{n}=\varphi_{n}\left(i y_{n}\right), \varphi_{n}(s)=\frac{s+\xi_{n}}{1+\xi_{n} s},\left|y_{n}\right| \leq r, \xi_{n} \rightarrow 1^{-} .
$$

Obviously, $\left|z_{n}\right|<1$. The pre-image $\varphi_{n}^{-1}(\Gamma)$ of the asymptotic path $\Gamma$ intersects the imaginary axis if $n$ sufficiently large. Hence we can find a subarc $C_{n}$ of $\mathbb{D} \cap \varphi_{n}^{-1}(\Gamma)$ such that $\operatorname{diam} C_{n} \geq \frac{1}{2}, \operatorname{Re} z>0$ for $z \in C_{n}$, and there exists a sequence $\left\{w_{n}\right\}$ in the $\operatorname{arc} \varphi_{n}\left(C_{n}\right) \subset \Gamma$ such that $w_{n} \rightarrow 1$ as $n \rightarrow \infty$. Then

$$
\max _{s \in C_{n}}\left|f\left(\varphi_{n}(s)\right)\right|=\max _{z \in \varphi_{n}\left(C_{n}\right)}|f(z)| \rightarrow 0(n \rightarrow \infty) .
$$

Since $f \circ \varphi_{n}$ is harmonic Bloch in $\mathbb{D}$ by Lemma 1 , we obtain from Theorem 2 that $f\left(\varphi_{n}(s)\right) \rightarrow 0$ as $n \rightarrow \infty$, uniformly in $|s| \leq r$. Hence (4.1) shows that $f\left(z_{n}\right) \rightarrow 0(n \rightarrow \infty)$.

From Theorem 3, we know that for harmonic Bloch mappings, asymptotic values and angular limits are identical. In the following, we can furthermore prove a result that applies also if there is no asymptotic values. 
Theorem 4. Let $f=h+\bar{g} \in \mathscr{B}_{\mathcal{H}}$ and $E \subset \mathbb{C}$. If

$$
\sup _{z \in \mathbb{D}}|\operatorname{Im} g(z)|<\infty \text { or } \sup _{z \in \mathbb{D}}|\operatorname{Im} h(z)|<\infty,
$$

and if $\Gamma$ is a Jordan arc that ends at $\xi \in \mathbb{T}$ and lies otherwise in $\mathbb{D}$ such that

$$
\limsup _{z \rightarrow \xi, z \in \Gamma} \operatorname{dist}[f(z), E]<\infty,
$$

then, for every Stolz angle $A$ with vertex $\xi$,

$$
\limsup _{z \rightarrow \xi, z \in A} \operatorname{dist}[f(z), E]<\infty \text {. }
$$

Choosing $E=\{|w| \leq r\}$ or $E=\mathbb{R}$ we see that if $|f(z)|$ or $\operatorname{Im} f(z)$ remains bounded as $z \rightarrow \xi$ along some arc $\Gamma$ with $\sup _{z \in \mathbb{D}}|\operatorname{Im} g(z)|<\infty$ or $\sup _{z \in \mathbb{D}}|\operatorname{Im} h(z)|<\infty$, then this is true in every Stolz angle.

This theorem is indeed a harmonic analog of [10, Theorem 9.5] (see also [1]) for Bloch functions.

Proof of Theorem 4. Without loss of generality, we assume that $\xi=1$ and $\sup _{z \in \mathbb{D}}|\operatorname{Im} g(z)|<\infty$. Suppose that (4.4) is false for some Stolz angle $A$ with vertex 1 . Then there exist $z_{n} \in A$ with

$$
\operatorname{dist}\left[f\left(z_{n}\right), E\right] \rightarrow \infty, z_{n} \rightarrow 1 \quad(n \rightarrow \infty),
$$

which is equivalent to

$$
\inf _{w \in E}\left|h\left(z_{n}\right)+g\left(z_{n}\right)-2 i \operatorname{Im} g\left(z_{n}\right)-w\right| \rightarrow \infty, z_{n} \rightarrow 1 \quad(n \rightarrow \infty) .
$$

As in the proof of Theorem 3, we write $z_{n}$ in the form of (4.1) and determine $\operatorname{arcs} C_{n} \subset \mathbb{D} \cap \varphi_{n}^{-1}(\Gamma)$ such that $\varphi_{n}\left(C_{n}\right) \rightarrow 1$ as $n \rightarrow \infty$. The function

$$
b_{n}(s)=\frac{1}{h\left(\varphi_{n}(s)\right)+g\left(\varphi_{n}(s)\right)-\left(h\left(z_{n}\right)+g\left(z_{n}\right)\right)}
$$

is meromorphic, and satisfies

$$
\left(1-|s|^{2}\right) \frac{\left|b_{n}^{\prime}(s)\right|}{1+\left|b_{n}(s)\right|^{2}} \leq\left(1-\left|\varphi_{n}(s)\right|^{2}\right)\left(\left|h^{\prime}\left(\varphi_{n}(s)\right)\right|+\left|g^{\prime}\left(\varphi_{n}(s)\right)\right|\right) \leq \alpha .
$$

It follows from (4.3), the inequalities $\sup _{z \in \mathbb{D}}|\operatorname{Im} g(z)|<\infty$ and

$$
\begin{aligned}
& \frac{1}{\left|h\left(\varphi_{n}(s)\right)+g\left(\varphi_{n}(s)\right)-\left(h\left(z_{n}\right)+g\left(z_{n}\right)\right)\right|} \\
& \leq \frac{1}{\left\|f\left(\varphi_{n}(s)\right)-w+2 i \operatorname{Im}\left(g\left(\varphi_{n}(s)\right)-g\left(z_{n}\right)\right)|-| f\left(z_{n}\right)-w\right\|}(w \in E)
\end{aligned}
$$

that $b_{n}(s) \rightarrow 0$ for $s \in C_{n}, n \rightarrow \infty$. Consequently, by [10, Theorem 9.2], $b_{n}(s) \rightarrow 0$ as $n \rightarrow \infty$ uniformly in $|s| \leq r$. This contradicts the fact that $b_{n}\left(i y_{n}\right)=\infty$ and $\left|y_{n}\right| \leq r$ by (4.1). 
In the remaining part of this section, we consider a method to associate to each harmonic mapping in the little harmonic Bloch space $\mathscr{B}_{\mathcal{H}, 0}$ another function $f^{*}$ in the harmonic Bloch space $\mathscr{B}_{\mathcal{H}}$ in such a way that $f$ has a finite angular limit where $f^{*}$ is locally bounded. The idea of the methods comes from the theory of the lacunary series, which was used in [4]. For completeness, we give the proof of the main result.

Now, we first introduce some polynomials. For the harmonic mapping

$$
f(z)=h(z)+\overline{g(z)}=\sum_{n=0}^{\infty} a_{n} z^{n}+\overline{\sum_{n=1}^{\infty} b_{n} z^{n}} \text { with } z \in \mathbb{D},
$$

we define polynomials $p_{0}(z)=a_{0}+a_{1} z+a_{2} z^{2}$ and

$$
p_{k}(z)=\sum_{n=2^{k-1}+2}^{2^{k}} \frac{2 n-2^{k}-2}{n-1} a_{n} z^{n}+\sum_{n=2^{k}+1}^{2^{k+1}} \frac{2^{k+1}-n+1}{n-1} a_{n} z^{n}
$$

for $k=1,2, \ldots$. Induction shows that

$$
\sum_{k=0}^{m} p_{k}(z)=\sum_{n=0}^{2^{m}} a_{n} z^{n}+\sum_{n=2^{m}+1}^{2^{m+1}} \frac{2^{m+1}-n+1}{n-1} a_{n} z^{n}
$$

and since $\lim \sup \left|a_{n}\right|^{1 / n} \leq 1$, it follows that

$$
h(z)=\sum_{k=0}^{\infty} p_{k}(z) \text { for } z \in \mathbb{D} .
$$

Similarly, we define polynomials $q_{0}(z)=b_{1} z+b_{2} z^{2}$ and

$$
q_{k}(z)=\sum_{n=2^{k-1}+2}^{2^{k}} \frac{2 n-2^{k}-2}{n-1} b_{n} z^{n}+\sum_{n=2^{k}+1}^{2^{k+1}} \frac{2^{k+1}-n+1}{n-1} b_{n} z^{n}
$$

for $k=1,2, \ldots$. We also have

$$
g(z)=\sum_{k=0}^{\infty} q_{k}(z) \text { for } z \in \mathbb{D} .
$$

Obviously, all $p_{k}$ and $q_{k}(k=1,2, \ldots)$ are in the class $H^{\infty}$ of bounded analytic functions $f$ in $\mathbb{D}$, which is a Banach space with the norm

$$
\|f\|_{\infty}=\sup _{z \in \mathbb{D}}|f(z)| \text {. }
$$

Now, we give the following three lemmas which is useful in the proof of next result.

Lemma 3 ([4, Proposition 1$])$. If $h \in \mathscr{B}$, then

$$
\left\|p_{k}\right\|_{\infty}=\sup _{|z| \leq 1}\left|p_{k}(z)\right| \leq 6\|h\|_{\mathscr{B}}
$$


for $k=0,1, \ldots$, and if $h \in \mathscr{B}_{0}$, then

$$
\left\|p_{k}\right\|_{\infty} \rightarrow 0 \text { as } k \rightarrow \infty .
$$

Lemma 4 ([4, Proposition 2]). If $\left\|p_{k}\right\|_{\infty}$ is bounded, then $h \in \mathscr{B}$ and

$$
\|h\|_{\infty} \leq 16 \sup _{k \geq 0}\left\|p_{k}\right\|_{\infty} .
$$

If $\left\|p_{k}\right\|_{\infty} \rightarrow 0$ as $k \rightarrow \infty$, then $h \in \mathscr{B}_{0}$.

Lemma $5\left(\left[4\right.\right.$, Proposition 3]). Let $h \in \mathscr{B}$. If $s_{k}(z)=p_{0}(z)+p_{1}(z)+\cdots+p_{k}(z)$ and $r_{k}=1-2^{-k}$, then

$$
\left|h\left(r_{k} z\right)-s_{k}(z)\right| \leq 30\|h\|_{\mathscr{B}} \text { for }|z| \leq 1 .
$$

For $g(z)$ and $q_{k}(z)(k=0,1, \ldots)$, we have similar results as the above three lemmas. More details for Lemmas 3 and 4, see also [11] or [12, Vol 1, p. 115].

Theorem 5. If $f \in \mathscr{B}_{\mathcal{H}, 0}$, then there is a harmonic mapping $f^{*} \in \mathscr{B}_{\mathcal{H}, 0} \subset \mathscr{B}_{\mathcal{H}}$ such that, for all $\xi \in \mathbb{T}$,

$$
\sup _{r}\left|f^{*}(r \xi)\right|<\infty \Rightarrow \lim _{r \rightarrow 1} f(r \xi) \text { exists } \neq \infty \text {. }
$$

This theorem generalize [4, Theorem] for Bloch functions to the case of harmonic Bloch mappings.

Proof of Theorem 5. Let $f \in \mathscr{B}_{\mathcal{H}, 0}$. It follows from Lemma 3 that there exist two decreasing sequences $\left\{\varepsilon_{k}\right\}$ and $\left\{\epsilon_{k}\right\}$ such that

$$
\left\|p_{k}\right\|_{\infty}<\varepsilon_{k}^{2} \text { and }\left\|q_{k}\right\|_{\infty}<\epsilon_{k}^{2}
$$

for $k=0,1, \ldots$, where $p_{k}$ and $q_{k}$ are given by (4.8) and (4.10), respectively. We define

$$
f^{*}(z)=h^{*}(z)+\overline{g^{*}(z)}=\sum_{k=0}^{\infty} p_{k}^{*}(z)+\overline{\sum_{k=0}^{\infty} q_{k}^{*}(z)},
$$

where $p_{k}^{*}(z)=\varepsilon_{k}^{-1} p_{k}(z)$ and $q_{k}^{*}(z)=\epsilon_{k}^{-1} q_{k}(z)$ for $k=0,1, \ldots$. This coincides with the expansion (4.9) and (4.11).

Since $\left\|p_{k}^{*}\right\|_{\infty}<\varepsilon_{k}$ and $\left\|q_{k}^{*}\right\|_{\infty}<\epsilon_{k}$ by (4.12), we conclude from Lemma 4 that $h^{*}, g^{*} \in \mathscr{B}_{0}$, and then $f^{*} \in \mathscr{B}_{\mathcal{H}, 0}$.

Writing $s_{k}^{*}=p_{0}+p_{1}^{*}+\cdots+p_{k}^{*}$ and $S_{k}^{*}=q_{0}+q_{1}^{*}+\cdots+q_{k}^{*}$, partial summations give

$$
\sum_{k=0}^{N} \varepsilon_{k} p_{k}^{*}(z)=\varepsilon_{N} s_{N}^{*}(z)+\sum_{k=0}^{N-1}\left(\varepsilon_{k}-\varepsilon_{k+1}\right) s_{k}^{*}(z)
$$

and

$$
\sum_{k=0}^{N} \epsilon_{k} q_{k}^{*}(z)=\epsilon_{N} S_{N}^{*}(z)+\sum_{k=0}^{N-1}\left(\epsilon_{k}-\epsilon_{k+1}\right) S_{k}^{*}(z)
$$


It follows that

$$
\begin{aligned}
f(r \xi)= & h(r \xi)+\overline{g(r \xi)}=\sum_{0}^{\infty} \varepsilon_{k} p_{k}^{*}(r \xi)+\overline{\sum_{0}^{\infty} \epsilon_{k} q_{k}^{*}(r \xi)} \\
= & \lim _{N \rightarrow \infty}\left(\sum_{0}^{N} \varepsilon_{k} p_{k}^{*}(r \xi)\right)+\lim _{N \rightarrow \infty}\left(\sum_{0}^{N} \epsilon_{k} q_{k}^{*}(r \xi)\right) \\
= & \lim _{N \rightarrow \infty}\left(\varepsilon_{N} s_{N}^{*}(z)+\sum_{k=0}^{N-1}\left(\varepsilon_{k}-\varepsilon_{k+1}\right) s_{k}^{*}(z)\right) \\
& +\lim _{N \rightarrow \infty}\left(\epsilon_{N} S_{N}^{*}(z)+\sum_{k=0}^{N-1}\left(\epsilon_{k}-\epsilon_{k+1}\right) S_{k}^{*}(z)\right) \\
= & \sum_{k=0}^{\infty}\left(\varepsilon_{k}-\varepsilon_{k+1}\right) s_{k}^{*}(r \xi)+\sum_{k=0}^{\infty}\left(\epsilon_{k}-\epsilon_{k+1}\right) S_{k}^{*}(r \xi) .
\end{aligned}
$$

Let now $\left|f^{*}(r \xi)\right|$ be bounded in $0 \leq r<1$ for some $\xi \in \mathbb{T}$. By Lemma 5 , we have

$\left|f^{*}(r \xi)-s_{k}^{*}(\xi)-\overline{S_{k}^{*}(\xi)}\right| \leq\left|h^{*}(r \xi)-s_{k}^{*}(\xi)\right|+\left|g^{*}(r \xi)-S_{k}^{*}(\xi)\right| \leq 30\left(\|h\|_{\mathscr{B}}+\|g\|_{\mathscr{B}}\right)$

for $r=1-2^{-k}$. It follows that $\left|s_{k}^{*}(\xi)+\overline{S_{k}^{*}(\xi)}\right|$ is also bounded in $k$. Since $\left|s_{k}^{*}(\xi)+\overline{S_{k}^{*}(\xi)}\right|$ is continuous in $0 \leq r<1$ for each $k$ and since $\varepsilon_{k}-\varepsilon_{k+1} \geq 0$ and $\epsilon_{k}-\epsilon_{k+1} \geq 0$, we easily deduce that

$$
f(r \xi)=\sum_{k=0}^{\infty}\left(\varepsilon_{k}-\varepsilon_{k+1}\right) s_{k}^{*}(r \xi)+\overline{\sum_{k=0}^{\infty}\left(\epsilon_{k}-\epsilon_{k+1}\right) S_{k}^{*}(r \xi)}
$$

is uniformly continuous in $0 \leq r<1$ and therefore has a limit as $r \rightarrow 1^{-}$.

We write the class of bounded harmonic mappings $f$ in $\mathbb{D}$ as $h^{\infty}$, which is a Banach space with the norm

$$
\|f\|_{\infty}=\sup _{z \in \mathbb{D}}|f(z)| .
$$

An immediate consequence of [6, Theorem 3] is that $h^{\infty} \subset \mathscr{B}_{\mathcal{H}}$, however it is well known that a bounded harmonic functions doesn't always belong to $\mathscr{B}_{\mathcal{H}, 0}$ (see [2, Section 3]). In [2], Attele proved that if $f \in H^{\infty}$, then $f^{\prime}\left(r e^{i \theta}\right)\left(1-r^{2}\right) \rightarrow$ 0 for almost all $\theta$ as $r \rightarrow 1^{-}$. By using similar argument, we can generalize this result to the case of harmonic mappings and obtain the following remark.

Remark 1. Suppose that $f=h+\bar{g} \in h^{\infty}$ is a harmonic Bloch mapping in $\mathbb{D}$, then

$$
\left|e^{i \theta} h^{\prime}\left(r e^{i \theta}\right)+\overline{e^{i \theta} g^{\prime}\left(r e^{i \theta}\right)}\right|\left(1-r^{2}\right) \rightarrow 0 \text { for almost all } \theta \text { as } r \rightarrow 1^{-} .
$$




\section{References}

[1] J. M. Anderson, J. Clunie, and Ch. Pommerenke, On Bloch functions and normal functions, J. Reine Angew. Math. 270 (1974), 12-37.

[2] R. Attele, Bounded analytic functions and the little Bloch space, Internat. J. Math. Math. Sci. 13 (1990), no. 1, 193-198.

[3] F. G. Avkhadiev and K. J. Wirths, Schwarz-Pick Type Inequalities, Birkhäuser, Basel, 2009.

[4] J. J. Carmona, J. Cufi, and Ch. Pommerenke, On the angular limits of Bloch functions, Publ. Mat. 32 (1988), no. 2, 191-198.

[5] SH. Chen, S. Ponnusamy, and X. Wang, Area integral means, Hardy and weighted Bergman spaces of planar harmonic mappings, Kodai Math. J. 36 (2013), no. 2, 313324.

[6] F. Colonna, The Bloch constant of bounded harmonic mappings, Indiana Univ. Math. J. 38 (1989), no. 4, 829-840.

[7] _ Bloch and normal functions and their relation, Rend. Circ. Mat. Palermo (2) 38 (1989), no. 2, 161-180.

[8] P. Duren, Harmonic Mappings in the Plane, Cambridge Univ. Press, New York, 2004.

[9] O. Lehto and K. I. Virtanen, Boundary behaviour and normal meromorphic functions, Acta Math. 97 (1957), 47-65.

[10] Ch. Pommerenke, Univalent Functions, Vandenhoeck \& Ruprecht, Göttingen, 1975.

[11] A. Zygmund, Smooth functions, Duke Math. J. 12 (1945), 47-76.

[12] _ Trigonometric Series, Cambridge Univ. Press, 1968.

JinJING QIAO

Department of Mathematics

HeBei UNIVERSITY

Baoding, Hebei 071002, P. R. China

E-mail address: mathqiao@126.com

Hongya Gao

Department of Mathematics

Hebei University

Baoding, Hebei 071002, P. R. China

E-mail address: ghy@hbu.cn 